\title{
Particle image velocimetry investigation of steady flow over a backward- facing step
}

\author{
Sharul Sham Dol ${ }^{1, a}$ \\ ${ }^{1}$ Petroleum Engineering Department, Curtin University, Sarawak Campus CDT 250, Miri Sarawak, 98009 Malaysia
}

\begin{abstract}
The backward-facing step (BFS) is a heuristic example, allowing for complex phenomena to arise in a simple geometry. Particle Image Velocimetry (PIV) investigations of mean-velocity distributions of backward-facing step flow with steady inlet condition were carried out and good agreement was obtained between current and previously published results for $50 \leq R e \leq 400$. This confirms that the current experimental capabilities can provide detailed and accurate velocity information. The flow behaviour downstream the step depends on the strength of separated shear layer, which the circulation depends on the bulk flow, recirculation zone length and vortex formation time. Since the vortex formation process is governed by the circulation flux convected along the wall layer from the step, for $R e \leq 400$, all of the circulation contained in the shear layer is drawn into the recirculation region. Thus, in a case where the shear layer characteristics are modified (e.g. in higher Reynolds number and unsteady flows), the balance of circulation is modified that would result in shedding.
\end{abstract}

\section{Introduction}

The backward-facing step (BFS) is a heuristic example, allowing for complex phenomena to arise in a simple geometry. The steady flow over a BFS has received much interest over the past decades (e.g. [1-6]), mainly due to the abundance of competing physical phenomena (e.g. internal flow separation, shear layer instability, boundary layer growth, flow recirculation, vorticity flow, vortex shedding and flow reattachment) of practical pertinence. The internal separated flow regions appear in many engineering and biomedical applications. Examples of these include flows in heat exchangers or through sudden expansions in pipe networks.

A BFS is also a suitable prototypical example for regions of flow recirculation-reattachment in physiological flows, such as in arterial bifurcation, stenoses and many biomedical devices including venous and urinary catheters, fluid transfer implants and artificial heart valves [7]. In these kinds of applications, the inlet flows are normally pulsatile and often characterized by zones of highly unstable and disturbed flows downstream of the step. Haidekker et al. [8] in their experimental studies used the BFS as an in-vitro model to examine the response of mammalian cells to spatial and temporal wall shear stress variations induced by disturbed flow and rapidly changing near-wall flow patterns. They showed that under pulsatile conditions, the BFS geometry causes local amplification of the shear stress in the recirculation region and that strong temporal gradients of the of flow in the BFS model also leads to a significant stimulation of endothelial cell growth. The flow pulsation induces hydrodynamic instabilities that result in flow unsteadiness downstream of the sudden expansion.

The flow over a BFS with steady inlet condition has been extensively studied both experimentally and numerically (e.g. [2, 5, 6, 9, 10, 11, 12, 13, 14, 15]). Armaly et al. [2] performed Laser-Doppler measurements of velocity distribution and reattachment length downstream of a BFS mounted in a 18:1 aspect ratio (step span/step height) channel for a wide range of Reynolds number, $70<R e<8000$. They reported that the length of the recirculating flow region in the immediate vicinity of the BFS (primary vortex) has strong, non-linear dependence on the Reynolds number. The primary recirculation length increases up to $R e \sim 1200$. An upper wall recirculation region (secondary vortex) appears for $R e>\sim 400$ due to the adverse pressure gradient created by the sudden expansion. The flow field downstream the step becomes unsteady due to the complex interaction between these primary and secondary vortices [6]. These vortices have different sizes and characteristics. While many questions remain, these basic flows are understood considerably better than the unsteady flows over a BFS.

Thus, the steady results will serve to verify the validity and reliability of the experimental set-up and technique used in this study. Moreover, a dynamic characterization of the strength (circulation) of the steady primary recirculation region, which has not been discussed in earlier work, provides a useful perspective

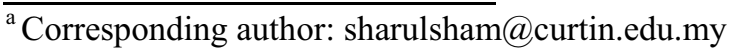


on how to handle the different flow parameters in a case where circulation must shed (e.g. turbulent, pulsatile flow). For this purpose, steady inlet flows for $50 \leq R e \leq$ 400 are experimentally investigated by using particle image velocimeter (PIV).

\section{Experimental apparatus and technique}

The experimental investigation was conducted in the BFS water channel as shown in Figure 1. This is a closed-loop flow chamber with a $250 \mathrm{~mm}$-long working section. It incorporates backward-facing step (BFS) with an expansion ratio $(H / h)$ of 2.0. The flow enters through a nozzle with an area contraction ratio of $10: 1$. This contraction was designed using the method of [16] to prevent over-speed separation. The outlet of the nozzle is connected to the inlet of the channel test section, which is $2 \mathrm{~mm}$ in height $(h), 50 \mathrm{~mm}$ in width $(W)$ and $100 \mathrm{~mm}$ in length up to the backward-facing step $(l)$. These dimensions ensure a two-dimensional fully developed flow at the cross-section where the step is located. The downstream length $(L)$ to the outlet of the channel is 150 $\mathrm{mm}$. The outlet channel is long enough to allow the flow recovering into a fully developed channel profile. This BFS model has an aspect ratio of $W / h=25$, which is sufficient to eliminate three-dimensional wall effects in the center of the span [17]. The channel walls were made of Plexi-glass for optical access with a laser measuring system. The laboratory coordinate system was taken with the origin centrally located at the sudden expansion plane. A Cartesian coordinate system is used with the $x$ axis aligned in the direction of the bulk flow, $U_{0}$, and the $y$-axis normal to the flow (transverse). A schematic of the BFS channel is shown in Figure $1(b)$.

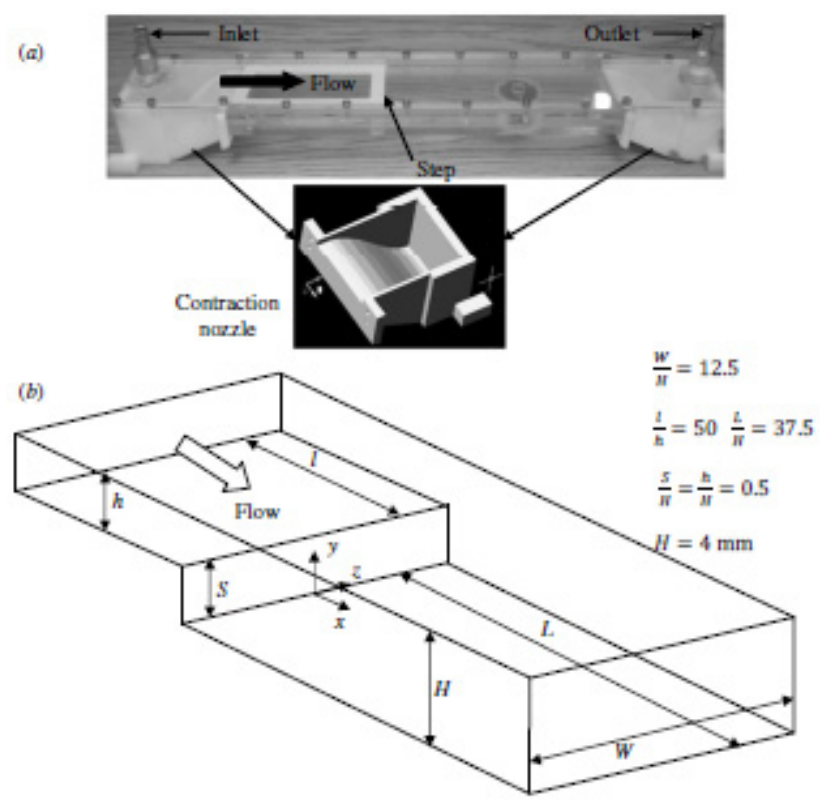

Figure 1. BFS channel; (a) Photograph of the channel; (b) Schematic of the BFS.

For steady flow experiments, a constant head gravity driven arrangement was selected, for which flow fluctuations could not be observed. Gravity driven flow for the steady flow experiments was achieved via hydrostatic pressure exerted by a difference in height between the supply reservoir and the BFS channel. Velocity measurements for steady flows were performed at $50 \leq R e \leq 400$ using high-frame-rate particle image velocimeter. The Reynolds number used in this study is defined based on $2 h$ (hydraulic diameter of the inlet channel) and average bulk velocity upstream of the channel, $U_{0}$.

Flow field information was obtained by using a LaVision FlowMaster high-frame-rate PIV system. The ability of the system to simultaneously give very high spatial resolution velocimetry over a relatively large flow region and time resolved flow fields makes the technique essential to the study of evolving dynamics of coherent structures. Silicon-carbide particles with $8 \mu \mathrm{m}$ nominal diameter were added to the water and used as tracers. The flow is illuminated by double-pulsed $\mathrm{Nd}$ :YLF laser (Photonics Industries DM20-527, $\lambda=527 \mathrm{~nm}$ ) with a maximum energy of $20 \mathrm{~mJ}$ per pulse per laser head at a repetition rate of $1000 \mathrm{~Hz}$.

In the present study the image recoding unit is a digital high-speed 10-bit CMOS camera of type HighSpeedStar 5 with maximum frame rate of $250 \mathrm{kHz}$. It has resolution of $1024 \times 1024$ pixels, captured at $3 \mathrm{kHz}$ frame rates and the pixel size is $17 \mu \mathrm{m} \times 17 \mu \mathrm{m}$. The camera was fitted with an AF micro-Nikkor $60 \mathrm{~mm}$ $\mathrm{f} / 2.8 \mathrm{D}$ lens and the object distance was adjusted to obtain a field-of-view of $\sim 18.4$ x $18.4 \mathrm{~mm}^{2}$. The spatial resolution was $0.0179856 \mathrm{~mm} /$ pixel. Single-frame mode with recording rate of $125-1000 \mathrm{~Hz}$ was used and 5001000 frames per acquisition were recorded in all the experiments. The vector analysis was done via DaVis 7.2 software in two steps; multi-pass with decreasingly smaller sizes interrogation windows of $64 \times 64$ and $16 \times$ 16 pixel were used in the first and second step, respectively to achieve more precise results and higher spatial resolution. An overlap of $50 \%$ of the interrogation area was used together with a Gaussian window function to minimize the loss of pairs. A detailed description of the technique and the uncertainty estimation can be found in [18-19].

\section{Results and discussions}

Results of PIV investigations of steady laminar flows over an expansion ratio 2:1 backward-facing step (BFS) at $50 \leq R e \leq 400$ are summarized here.

\subsection{Mean flow}

Figure $2(a-d)$ shows the mean vector flow fields and streamlines patterns for $50 \leq R e \leq 400$. The flow is laminar and steady. The results yield the expected primary zone of recirculating flow attached to the backward-facing step and also show no additional regions of flow separation downstream of the sudden expansion. This is consistent with the results reported in previous studies (e.g. [2, 4]. Armaly et al. [2] found that the secondary recirculation region (secondary vortex), due to an adverse pressure gradient created by the sudden expansion, on the opposite wall from the step develops for $R e>\sim 400$. 

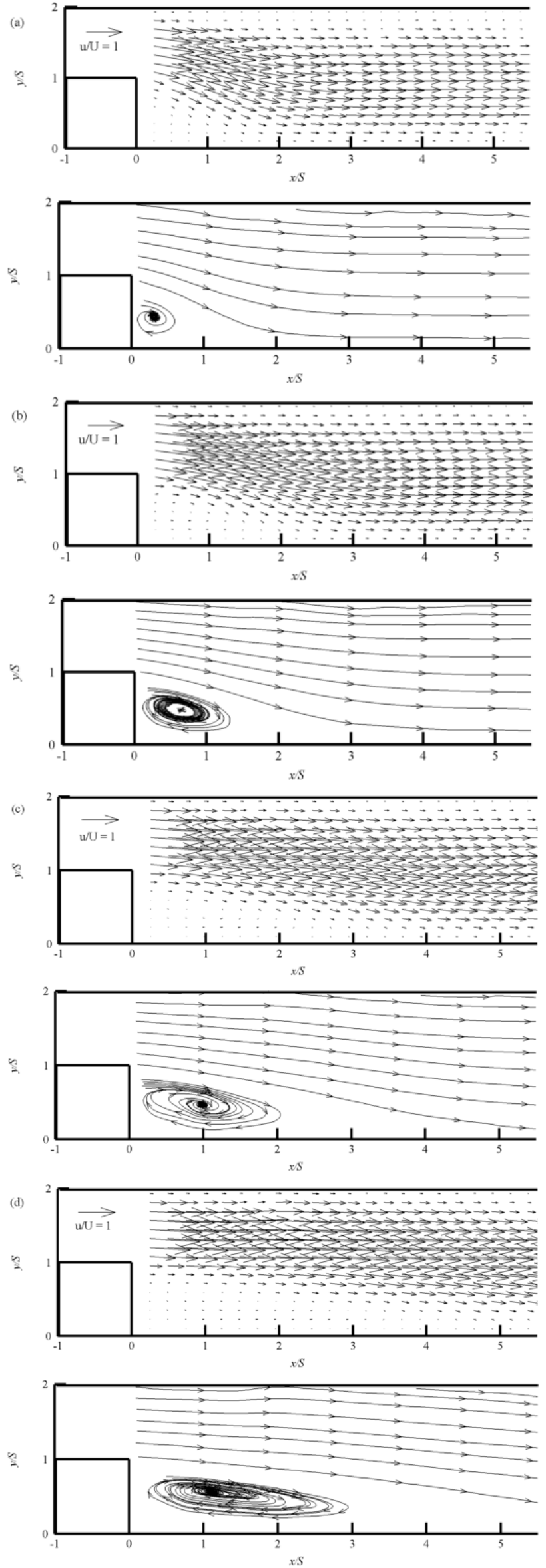

Figure 2. Mean flow fields and streamlines patterns; $(a) R e=$ 50; (b) $\operatorname{Re}=100$; (c) $\operatorname{Re}=200$; (d) $\operatorname{Re}=400$.
Velocity profiles as shown in Figure $3(a-d)$ demonstrate that the length of flow development downstream of the step increases with the Reynolds number. For $R e=50$ and 100 , the velocity profiles at $x / S$ $=4.5$ show that the velocity fields are close to that of a fully developed channel flow with a parabolic profile. However, deviations from the parabolic profile are present for higher Reynolds numbers $(R e=200$ and 400) indicating the flow is still redeveloping at $x / S=4.5$ due to a stronger separated shear layer, hence farther reattachment point.
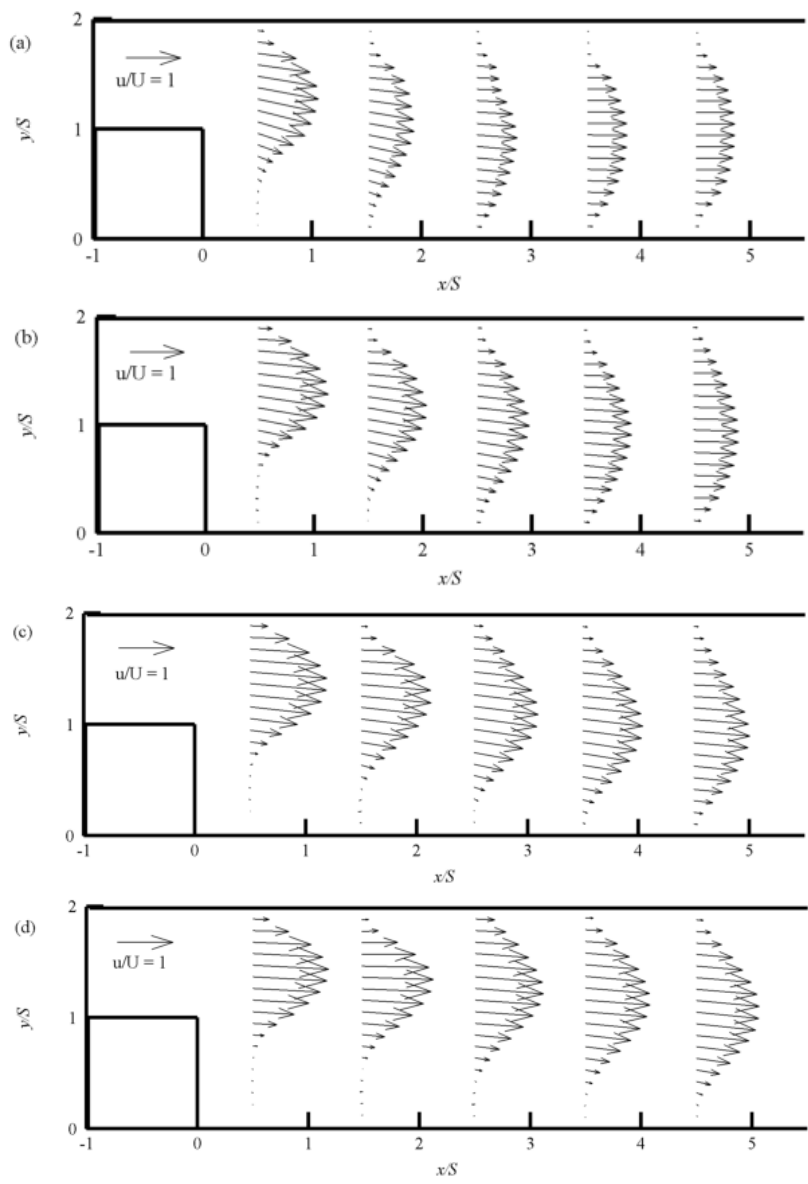

Figure 3. Velocity profiles at several locations downstream the sudden expansion; (a) $R e=50$; (b) $R e=100$; (c) $R e=200$; (d) $R e=400$

\subsection{Recirculation zone}

Figure 4 shows the variation of recirculation zone lengths, $X_{\mathrm{R}}$, with Reynolds numbers, $R e$. The results show that $X_{\mathrm{R}}$ increases almost linearly with $R e$ for $50 \leq$ $R e \leq 400$. The present results of $X_{\mathrm{R}}$ are consistent with the previous investigations $[2,14,20]$. Experimental results of [4] showed that the flow downstream of the step showed destabilization traits (e.g. transverse oscillations of the separated streamline) as soon as the secondary separation region appeared $(R e \sim 400-500)$. The highly energetic shear layer becomes unstable which breaks, leading to the formation of isolated convective vortices [15] and for a steady flow over a BFS, this phenomena can only be seen for $R e>\sim 1500$ [6]. The nearly linear increase of $X_{\mathrm{R}}$ with $R e$ suggests that the size 
of the recirculation region scales directly with the circulation flux along the separated shear layer as discussed in the next section.

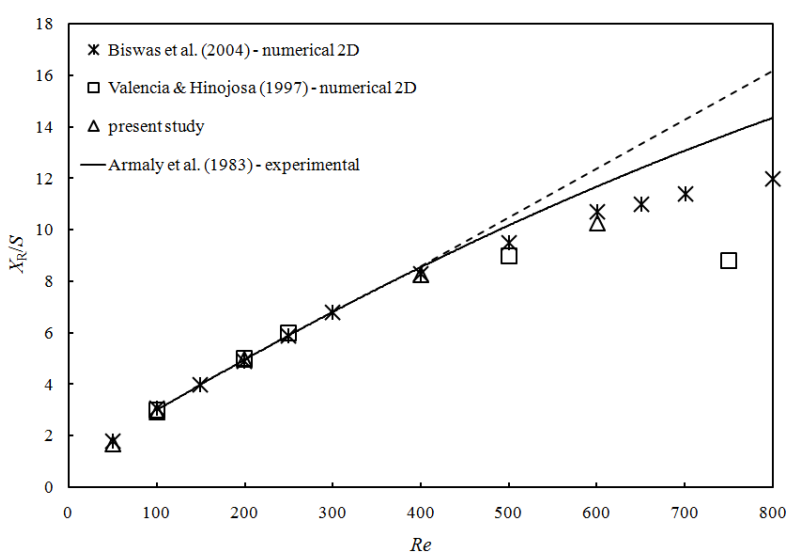

Figure 4. Variations of primary recirculation zone lengths with Re.

\subsection{Vortex strength and formation time}

Figure 5 shows the amount of circulation in the primary recirculation region (bound vortex) for $50 \leq R e \leq 400$. No vortex shedding occurs at this range of Reynolds numbers (i.e. there is no net convection of circulation downstream). The circulation flux must be balanced locally inside the steady recirculation region by the vorticity flux at the walls. Hence, it will be a good indication to see how the primary vortex characteristics (e.g. strength, formation time) changes with the bulk flow. The vortex strength (circulation), $\Gamma_{\mathrm{P}}$ was estimated according to vorticity integrated area of the primary vortex,

$$
\Gamma_{P}=\oint \vec{\omega} \cdot d \vec{A} \approx \sum_{i, j}(\omega)_{i, j} \Delta A_{i, j}
$$

where $\omega$ is the local vorticity, $i$ and $j$ are spatial indices, and $A$ is the vortex area as defined by [21]. The circulation $\frac{\Gamma_{P}}{U_{0} D}$ increases linearly, indicating that the strength of the primary recirculation region is linearly in proportion with $U_{0}$. The non-dimensional magnitude of the vorticity $\left(\omega=\frac{d v}{d x}-\frac{d u}{d y}\right)$ in the separated shear layer does not vary significantly with the Reynolds numbers (Figure 6), so that the circulation scales with the characteristic length $X_{\mathrm{R}}$.

The rate of circulation feeding the primary vortex is assumed to be the rate at which circulation is advected along the wall boundary layer separating at the step:

$$
\frac{D \Gamma}{D t} \approx \int u \frac{\partial u}{\partial y} d y=\frac{U_{1}{ }^{2}-U_{2}{ }^{2}}{2}
$$

where $U_{1}$ and $U_{2}$ are the velocities at the edges of the shear layer, the strength of which is $\Delta U=U_{1}-U_{2}$. Assuming that the shear layer behaves as a vortex sheet (no net vorticity flux from the shear layer), the initial circulation flux is preserved and should correspond to the circulation flux along the wall layer separating at the step (i.e. the wall no longer acts as a vorticity flux source). Hence, $U_{1}=k U_{0}(k=1.5$ for a laminar flow $)$ and and $U_{2}$ $=0$ such that:

$$
\frac{D \Gamma}{D t}=\frac{k^{2} U_{0}^{2}}{2}
$$

which is shown in Figure 7. It shows the flux scales with $U_{0}^{2}$.

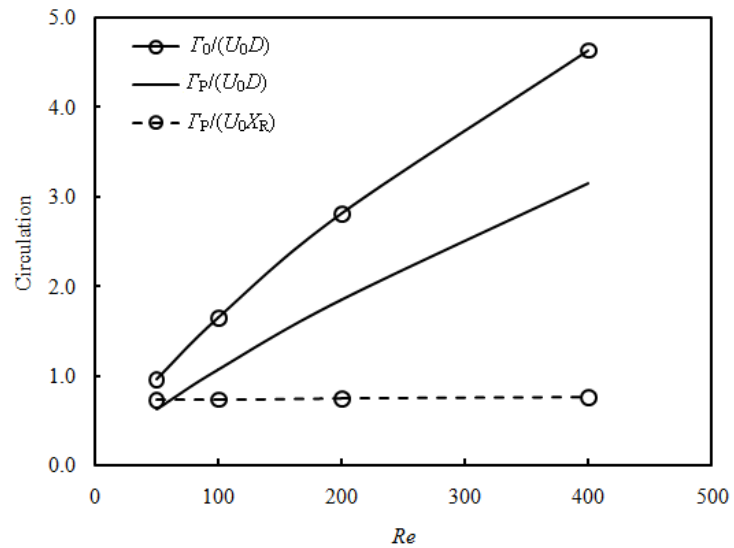

Figure 5. Variations of circulation with $R e$.
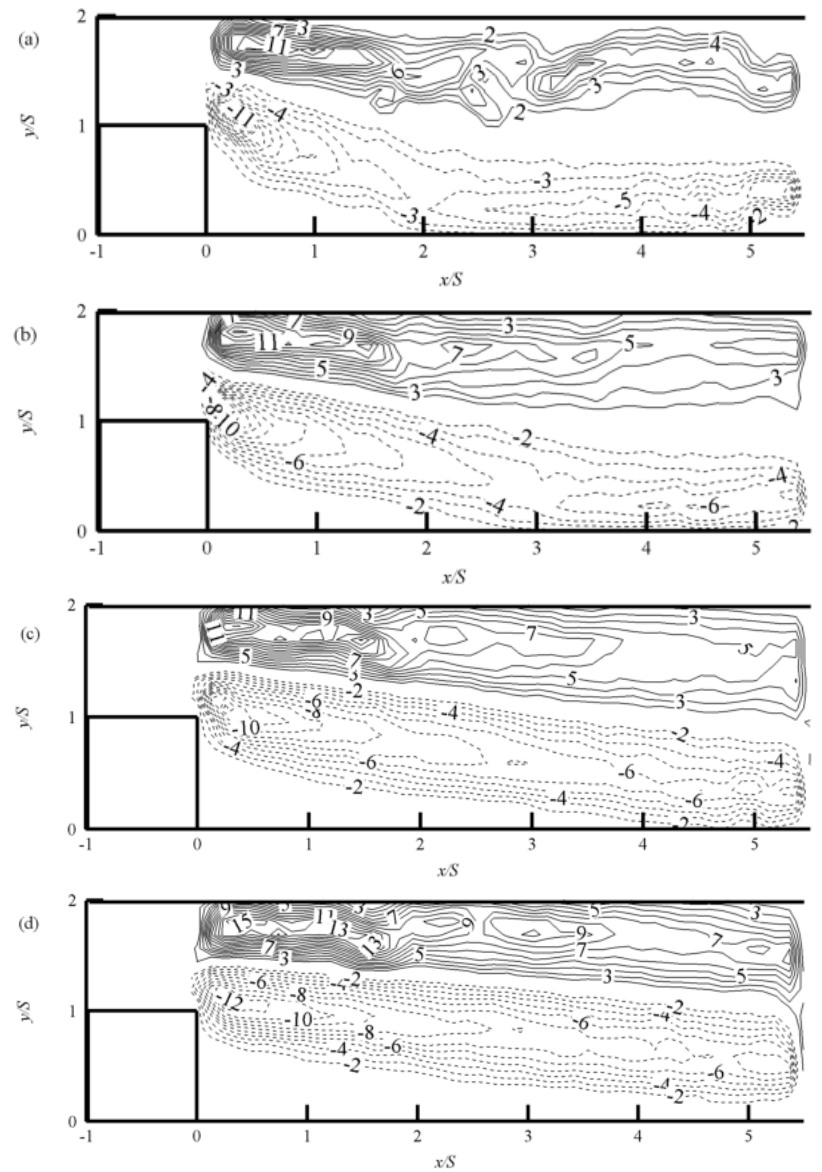

Figure 6. Mean vorticity contours $\left(\omega D / U_{0}\right)$. Dashed (solid) lines represent constant negative (positive) vorticity values; (a) $R e=50$; (b) $R e=100$; (c) $\operatorname{Re}=200$; (d) $R e=400$. 


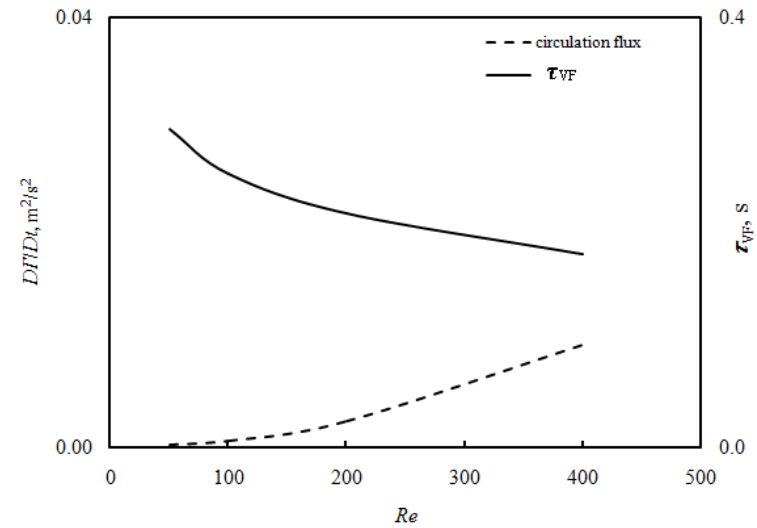

Figure 7. Variations of circulation flux and vortex formation time with $R e$.

If the shear layer is assumed to behave like a vortex sheet of intensity $\Delta U$, then the total circulation contained in the shear layer should be:

$$
\Gamma_{0}=\int \Delta u d s
$$

where $s$ is the distance from the step along the vortex sheet. The distance $s$ must scale with $X_{\mathrm{R}}$ such that $\Gamma_{0} \propto \Delta u X_{R}=k U_{0} X_{R}$. Assuming that the circulation losses at the walls (vorticity flux along the walls due to the wall-parallel pressure gradient) also scale with $U_{0}^{2}$, one obtain that

$$
\frac{\Gamma_{P}}{U_{0} X_{R}} \propto \frac{\Gamma_{0}}{U_{0} X_{R}}=\text { constant }
$$

This assumption appears at least approximately valid for $R e \leq 400$, as shown in Figure 5, where $\frac{\Gamma_{P}}{U_{0} X_{R}} \approx 0.75$. This observation confirms that the formation process is governed by the circulation flux convected along the wall layer from the step. The implication of this observation is that for $R e \leq 400$, all of the circulation contained in the shear layer is drawn into the recirculation region. Noting that $X_{\mathrm{R}}$ in this range increases almost linearly with $U_{0}$, it also implies that $\Gamma_{\mathrm{P}}$ should scale with $U_{0}^{2}$.

As the Reynolds number increases beyond 400, it is noted that the rate of growth of $X_{\mathrm{R}}$ decreases significantly with $R e$ (i.e. deviates from a linear progression), but the rate at which circulation is advected along the shear layer must continue to scale with $U_{0}^{2}$ (since the parabolic profile upstream of the step is independent of $R e$ for laminar flow). Again, assuming that the wall vorticity flux scales with $U_{0}^{2}$, which seems reasonable since the vortex is vertically bound by the shear layer, an equilibrium with the circulation contained in the vortex cannot be sustained. In other words, some of the circulation in the shear layer must be advected downstream. This loss of circulation would thus result in perturbation of the downstream flow resulting in the observed instabilities $[6,11,15]$. For example, in case where the recirculation region may become unstable due to the breakdown of shear layer that leads to the formation of shed vortices, as described by [15], the balance of circulation maybe interrupted because the excess of circulation is released during the shedding period. Armaly et al. [2] found that the recirculation zone length, $X_{\mathrm{R}}$ decreased in the range of $1200 \leq R e \leq 6600$ (transitional regime). This infers an abruption to a steady vortex formation.

Another observation from the foregoing analysis is that there is a natural time-scale associated with the formation of the primary vortex. This vortex formation time, $\tau_{\mathrm{VF}}$, can be simply defined as

$$
\tau_{\mathrm{VF}}=\frac{\Gamma}{\frac{D \Gamma}{D t}} \sim \frac{x_{R}}{U_{0}}
$$

which is shown in Figure 7. This formation time may play a role in unsteady and pulsating flows. If the frequency of pulsation is very low (period of pulsation, $T$, much longer than $\tau_{\mathrm{VF}}$ ), it is expected that the influence of the pulsation will be small. Conversely, if $T \ll \tau_{\mathrm{VF}}$, the pulsation will interfere with the vortex formation [18-19]. Examples of two cases of pulsatile flows with different period of pulsation are given below to see the effects of this vortex formation time.

The mean streamlines patterns for $S t=f D / U_{0}=0.4$ where $f$ is the pulsation frequency and for $R e=100$ is shown in Figure 8. The inlet pulsation leads to different flow behaviour downstream of the sudden expansion compared to the steady flow field. The main different between the mean flow for the pulsatile and steady cases is the appearance of the upper wall recirculation region (secondary vortex). This upper wall separation only happens at $R e>\sim 400$ for the steady case. The upper wall vortex leads to a destabilization in the flow field through concave curvature instability of the boundary layer $[6,18$, 19 ] and thus it is expected the circulation will be shed.

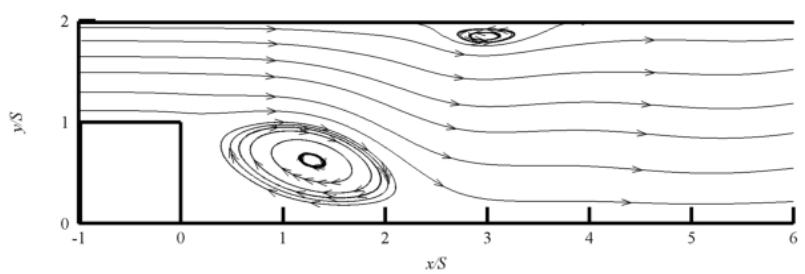

Figure 8. Mean streamlines patterns $(R e=100$ and $S t=0.4)$.

Although the average Reynolds number for $S t=0.4$ is the same to the steady case $(R e=100)$, the mean velocity profiles show differences, as shown in Figure 9. The streamwise velocity profile at $x / S=1$ (Figure $9 a$ ) shows that the flow inside the primary recirculation region (backflow) has higher velocity magnitude, implying the vortex has greater circulation for the pulsatile case. The streamwise velocity profile at $x / S=5$ for the steady flow shows that the velocity fields are close to that of a fully developed channel (parabolic profile) whilst the flow is still redeveloping for the pulsatile case (Figure $9 b$ ). The transverse velocity profiles also indicate the deviations from the steady flow behaviour (Figure 9c). These differences can be attributed to modification to the momentum transfer due to the flow pulsatility.

For $S t=0.035$, the formation of primary and secondary vortices is regular but no shedding is observed. Although the forming primary and secondary vortices are much stronger (e.g. compared to $S t=0.4$ ), these remain 
bound during the cycle and both the primary and secondary vortices lose their strength as soon as they enter the deceleration phase and eventually disappeared at the end of the pulsation cycle. Pulsatile flow with very small Strouhal number behaves more like a steady flow [22].
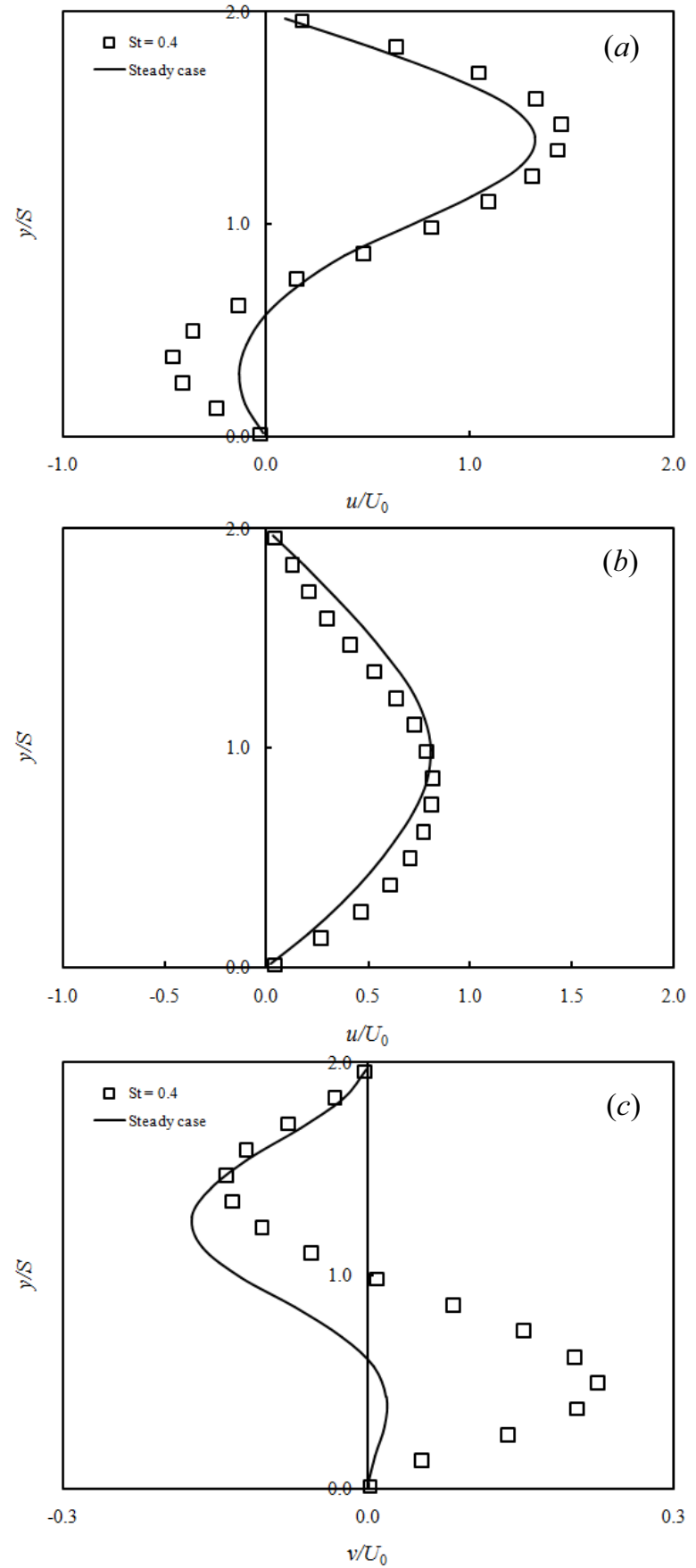

Figure 9. Mean velocity profiles for $R e=100 ;(a)$ Streamwise, $x / S=1 ;(b)$ Streamwise, $x / S=5 ;(c)$ Transverse, $x / S=1$.

\section{Conclusion}

PIV investigations of mean-velocity distributions of backward-facing step flow with steady inlet condition were carried out by the author, and good agreement was obtained between current and previously published results for $50 \leq R e \leq 400$. This confirms that the current experimental capabilities can provide detailed and accurate velocity information. The flow behaviour downstream the step depends on the strength of separated shear layer, which the circulation depends on the bulk flow, $U_{0}$, recirculation zone length, $X_{\mathrm{R}}$, and vortex formation time, $\tau_{\mathrm{VF}}$. Since the vortex formation process is governed by the circulation flux convected along the wall layer from the step, for $R e \leq 400$, all of the circulation contained in the shear layer is drawn into the recirculation region. Thus, in a case where the shear layer characteristics are modified (e.g. in higher Reynolds number or unsteady flow), the balance of circulation is modified that would result in shedding.

\section{References}

1. R. J. Goldstein, V. L. Eriksen, R. M. Olson, E. R. G. Eckert, Laminar separation reattachment, and transition of flow over a downward-facing step, Trans. ASME D: J. Basic Engng. 92, 732 (1970).

2. B. F. Armaly, F. Durst, J. C. F. Pereira, B. Schönung, Experimental and theoretical investigation of backward-facing step flow, J. Fluid Mech. 127, 496 (1983).

3. K. B. Chun, H. J. Sung, Visualization of a locallyforced separated flow over a backward-facing step, Exp. Fluids 25, 133 (1998).

4. T. Lee, D. Mateescu, Experimental and numerical investigation of 2D backward-facing step flow, $J$. Fluids Struct. 12, 703 (1998).

5. J. Kostas, J. Soria, M. S. Chong, Particle image velocimetry measurements of a backward-facing step flow, Exp. Fluids 33(6), 838 (2002).

6. H. P. Rani, W. H. S. Tony, S. F. T. Eric, Eddy structures in a transitional backward-facing step flow, J. Fluid Mech. 588, 43 (2007).

7. G. A. Truskey, K. M. Barber, T. C. Robey, L. A. Olivier, M. P. Combs, Characterization of a sudden expansion flow chamber to study the response of endothelium to flow recirculation, ASME J. Biomech. Engg. 117, 203 (1995).

8. M. A. Haidekker, C. R. White, J. A. Frangos, Analysis of temporal shear stress gradients during the onset phase of flow over a backward-facing step, J. Biomech. Engg. 123, 455 (2001).

9. J. Kim, and P. Moin, Application of a fractional step method to incompressible flows, J. Comput. Phys. 59, 308 (1985).

10. I. Grant, E. Owens, Y. Yan, Particle image velocimetry measurements of the separated flow behind a rearward facing step, Exp. Fluids 12, 238 (1992).

11. P. T. Williams, A. J. Baker, Numerical simulations of laminar flow over a 3D backward-facing step, Int. J. Numer. Methods Fluids 24, 1159 (1997).

12. T. P. Chiang, T. W. H. Sheu, A numerical revisit of backward-facing step flow problem, Phys. Fluids 11(4), 874 (1999).

13. D. Barkley, M. G. M Gomes, and Henderson, R. D. Three-dimensional instability in flow over a 
backward-facing step, J. Fluid Mech. 473, 190 (2002).

14. G. Biswas, M. Breuer, F. Durst, Backward-facing step flows for various expansion ratios at low and moderate Reynolds numbers, J. Fluids Eng. 126, 362 (2004)

15. C. P. Schram, C., P. Rambaud, M. L. Riethmuller, Wavelet based eddy structure eduction from a backward facing step flow investigated using particle image velocimetry, Exps. Fluids 36, 233 (2004).

16. P. A. Libby, H. R. Reis The design of twodimensional contraction sections, Quarterly of Applied Mathematics 10, 95 (1951)

17. V. de Brederode, P. Bradshaw, Three-dimensional flow in nominally two-dimensional separation bubbles, I. Flow behind a rearward-facing step, Aero Report 72-19, Imperial College of Science and Technology, London (1972).

18. S. S. Dol, M .M. Salek, R. J. Martinuzzi, Salek, R. J. Martinuzzi, Effects of Pulsation to the Mean Field and Vortex Development in a BackwardFacing Step Flow. Journal of Fluids Engineering, 136(1) (2014).

19. S. S. Dol, M .M. Salek, R. J. Martinuzzi, Energy Redistribution Between the Mean and Pulsating Flow Field in a Separated Flow Region. Journal of Fluids Engineering, 136(11), 111105 (2014).

20. A. Valencia, L. Hinojosa, Numerical solutions of pulsating flow and heat transfer characteristics in a channel with a backward-facing step, Heat and Mass Transfer 32,143 (1997).

21. J. Jeong, F. Hussain, On the identification of a vortex, J. Fluid Mech. 285, 69 (1994).

22. M .M. Salek, S. S. Dol, R. J. Martinuzzi, Analysis of Pulsatile Flow in a Separated Flow Region, ASME Proceedings 5th Symposium on Fundamental Issues and Perspectives in Fluid Mechanics, pp. 1429-1438 (2009). 